\title{
TEXTURES OF SILVER-HALIDE INFRARED FIBRES
}

\author{
W. BÖCKER, H. G. BROKMEIER and H. J. BUNGE
}

Department of Physical Metallurgy Technical University of Clausthal, Germany

(Received 10 May 1995)

Fibres consisting of a silver chloride core, a silver bromide cladding and a metallic silver coating were produced by co-extrusion followed by wire drawing and annealing. In most cases the core material was initially single crystalline. Together with the different flow stresses of core and cladding this may lead to inhomogeneous, non-axially symmetric flow. Particularly it may lead to a non-circular shape of the boundary between core and cladding which must be avoided when the fibres are to be used as infrared light conductors.

The deviation from axially symmetric flow was studied with the help of texture investigations using neutron diffraction. The high penetration depth of neutrons allows the simultaneous measurement of the textures of all three phases of the composite fibres. Using neutron diffraction texture analysis as a sensitive flow indicator, coated "core-cladding" as well as "graded" fibres with sufficient axial symmetry could thus be obtained. The fibres are well suited as infrared light conductors.

KEY WORDS Silver halide fibres, infrared light conductors, co-extrusion, wire drawing, neutron diffraction texture measurement

\section{INTRODUCTION}

Optical fibres for the mid-infrared region (MIR-fibres) have been of increasing interest for several applications. Mainly in the fields of infrared spectroscopy and high power laser delivery in medical and industrial applications, progress in the fabrication of these fibres has been reported in recent years (Artjushenko et al. 1986, 1991). Among others, crystalline silver halide materials are the most promising materials for these optical fibres. Silver halides have plastic properties very similar to those of metals. Hence, they have often been used as model substances for the plastic behaviour of metals ("transparent metals"). Particularly, texture studies in silver halides have been done in analogy to texture formation in metals (Nye 1949, Frommeyer and Wassermann 1976). This similarity can thus also be used for the production of optical fibres by methods which are well introduced in the production of metal wires. In order to obtain good wave-guide properties, internal total reflection must be achieved either by a discontinuity or a continuous gradient of the refraction index. In the system of $\mathrm{AgCl}-\mathrm{AgBr}$ this can be achieved by using a core-cladding structure with different compositions within the solid solution range or by a continuous variation of composition, respectively. Several techniques have been suggested in the literature, how to produce core-cladding fibres (Artjuskenko et al. 1986, 1991). 
Taking the similarity with metals into account, metallurgical techniques of extrusion and wire drawing must be considered as highly promising. Particularly, techniques of co-deformation of core-cladding composites are well known metallurgical techniques. Finally, the core-cladding fibre must be given an external coating in order to prevent it from mechanical damage. Also, such coating is highly desirable in order to protect the photo-sensitive material from uv-light. An external coating must not react chemically with the silver halide. From this point of view and because of the similarity of plastic properties, metallic silver is a favorite candidate for an external coating. Hence, a production technology of three-layer fibres by "metallurgical" techniques seems to be most promising.

The analogy between silver halides and metals can also be extended to the well established control of plastic deformation by texture studies. Hence, it was the purpose of the present paper to follow the production process of two- and three-layer composite fibres by texture measurements.

\section{PRODUCTION OF THE FIBRES}

In the present study, core-cladding fibres were produced by co-extrusion as is illustrated schematically in Figure 1. In order to obtain highest optical properties a single crystalline $\mathrm{AgBr}$ preform had to be used for the core. It was cladded by a polycrystalline $\mathrm{AgCl}$ mantle. This composite preform was then extruded with deformation degrees in the range of $\eta \sim 93 \%$. The extruded rod was inserted into a silver tube and was cold drawn with further deformation degrees in the range of $\eta=91 \%$. In some cases the

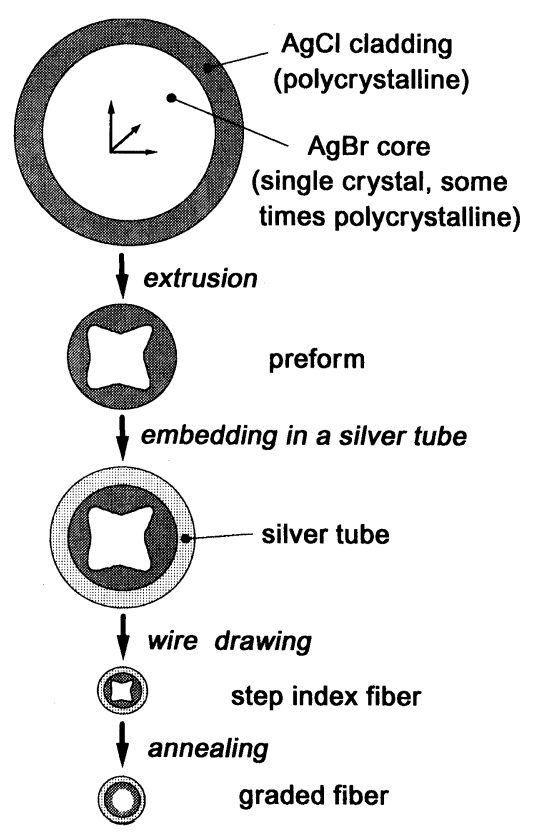

Figure 1 Production scheme of composite fibres $\mathrm{Ag}-\mathrm{AgC1}-\mathrm{AgBr}$. 
so obtained three-layer fibre was annealed which lead to recrystallization of the deformed structure. Also in some cases diffusion annealing was used which leads to a continuous variation of composition, in the solid solution range $\mathrm{AgCl}-\mathrm{AgBr}$. Starting diameters of $30 \mathrm{~mm}$ were thus reduced to final diameters in the range $3 \mathrm{~mm}$.

Co-extrusion of the core-cladding preform of Figure 1 may lead to inhomogeneous deformation with a complex stress-strain field according a minimum deformation work condition. Thereby the following influencing parameters must be considered:

1) The flow-stress (hardness) of the $\mathrm{AgCl}$ cladding is different from that of the $\mathrm{AgBr}$ core.

2) The $\mathrm{AgCl}$ cladding was used in a polycrystalline state which leads to a higher flow stress compared with a single crystalline material.

3) The single crystalline core has strongly anisotropic mechanical properties. Also the polycrystalline (textured) cladding material has, in principle, anisotropic properties although to a much lesser degree than the single crystalline core.

4) Besides on the flow stresses, the flow conditions also depend on the diameters of the different layers shown schematically in Figure 2.

The inhomogeneous flow field, in turn, has two consequences:

1) It leads to a non-circular deformation of the originally circular interface between core and cladding. This is a most undesirable effect because it leads to a deterioration of the optical properties. Hence, it was one of the most important tasks of the present study to find conditions how to avoid this effect.

2) An inhomogeneous flow field leads to an inhomogeneous orientation change of the originally single crystalline core material. At higher deformation degrees the initial single crystalline state thus becomes polycrystalline. Hence, two different effects must be expected:

2a) An inhomogeneous texture, i.e. texture field, is developed, mainly in the core but possibly also in the cladding.

2b) The global texture i.e. the volume average over the local textures, will not be axially symmetric although the total deformation of the whole composite fibre is axially symmetric.

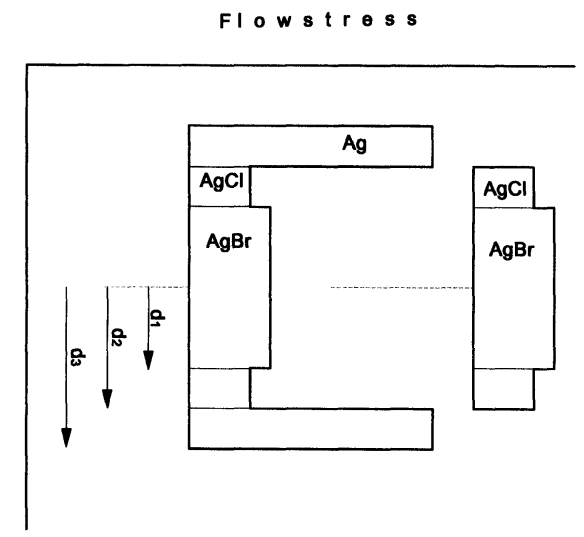

Figure 2 Diameters and relative flow stresses of the materials forming the composite fibre. 
Hence, texture studies are very well suited to obtain insight into the flow conditions of a complex multiphase structure as shown in Figure 1 with the goal to produce a cylindrically symmetric distribution of the refractive index.

The deviation from the circular shape of the interface boundary between $\mathrm{AgBr}$ core and $\mathrm{AgCl}$ cladding in Figure 1 can be reduced by:

1) Using small cladding thickness.

2) Using core materials with axially symmetric plastic properties.

The first principle is limited by the requirement that the thickness of the cladding in the final state must still be greater than the wavelength of the used light, i.e. $>10 \mu \mathrm{m}$. The second principle can be achieved, for instance, by using polycrystalline preforms in the core, as well. This is advantageous from the "mechanical" point of view but it was disadvantageous from the optical point of view. Hence, mainly single crystalline preforms were used for the core.

In the present paper we deal mainly with the plastic properties during extrusion and cold drawing of the composite fibres. Nevertheless, it is the purpose of the investigations to achieve good optical properties and to find conditions, how fibres with good mechanical as well as optical properties could be obtained.

\section{EXPERIMENTAL INVESTIGATIONS}

Texture investigations can be done by $\mathrm{X}$-ray or neutron diffraction. Table 1 compares the linear absorption coefficients for two different $\mathrm{X}$-ray wavelengths and neutrons $(\lambda=1.33 \AA$ ) as well as the corresponding penetration depths in the three used materials. These values must be compared with the diameters of the samples to be studied, examples of which are given in Table 2. Furthermore, it was necessary to measure the textures of all three layers with references to the same sample coordinate system. Hence, it was virtually impossible to separate the three layers and to measure their textures individually. Rather, simultaneous texture measurements had to be done. According to the values of Table 1,2 this could only be achieved by neutron diffraction.

Because of the lower scattering cross section, neutron diffraction requires bigger sample volumes compared with X-ray diffraction. In the present study samples of relatively small diameters (from the standpoint of neutron diffraction) had to be investigated. In such cases the sample volume can be increased by using bundles of wires. This method seemed, however, not appropriate in the present case since it had

Table 1 Linear absorption coefficients $\mu$ and the corresponding penetration depths $d=\mu^{-1}$ for the three considered materials, for two $X$-ray wavelengths and for neutrons $\lambda=1.33 \AA$ (see e.g. Sagel 1958).

\begin{tabular}{ccccccc}
\hline & \multicolumn{3}{c}{$\mu\left[\mathrm{cm}^{-1}\right]$} & & $d[\mu \mathrm{m}]$ & \\
\hline & $\mathrm{CuK} K_{\alpha}$ & MoK $_{\alpha}$ & Neutron & $C u K_{\alpha}$ & MoK & Neutron \\
\hline $\mathrm{Ag}$ & 2339 & 300.0 & 2.493 & 4.27 & 33.3 & 4011 \\
$\mathrm{AgC1}$ & 1077 & 135.9 & 1.802 & 9.28 & 73.6 & 5548 \\
$\mathrm{AgBr}$ & 1084 & 332.8 & 1.089 & 9.22 & 30.05 & 9186 \\
\hline
\end{tabular}


Table 2 Characteristics of the samples. Dimensions, sample volume, phases, and volume fractions

\begin{tabular}{lllll}
\hline Sample & Phase & Diameter & Thickness & Vol. fraction \\
\hline Extruded & total & $7.6 \mathrm{~mm}$ & - & 1.0 \\
& $\mathrm{AgBr}$ & $5 \mathrm{~mm}$ & - & 0.42 \\
& $\mathrm{AgC1}$ & - & $2.5 \mathrm{~mm}$ & 0.58 \\
\hline \multirow{2}{*}{ Cold drawn } & total & $3 \mathrm{~mm}$ & - & 1.0 \\
& $\mathrm{AgBr}$ & $2.63 \mathrm{~mm}$ & - & 0.77 \\
& $\mathrm{AgC1}$ & - & $0.06 \mathrm{~mm}$ & 0.03 \\
& $\mathrm{Ag}$ & - & $0.31 \mathrm{~mm}$ & 0.20 \\
\hline Annealed & total & $3 \mathrm{~mm}$ & - & 1.0 \\
& $\mathrm{AgBr}$ & $2.51 \mathrm{~mm}$ & - & 0.70 \\
& $\mathrm{AgBr} / \mathrm{AgC1}-$ & - & $0.18 \mathrm{~mm}$ & 0.10 \\
& $\mathrm{Gradient}$ & - & $0.31 \mathrm{~mm}$ & 0.20 \\
\hline
\end{tabular}

to be guaranteed that the individual wire sections were not rotated with respect to each other about their axes. Therefore, individual cylindrical samples were used with diameters as specified in Table 2. It is seen that the absolute volumes of the phases in which textures had to be measured are relatively small compared with the optimum standard volume. Furthermore, also the volume fractions of some of the phases (within the sample) are small in some cases. Hence, relatively long measuring times had to be used and, for the smaller volume fractions, the intensity-to-background ratio was low.

Furthermore, because of the three-phase structure, peak overlap had to be taken into consideration. Table 3 summarizes the essential crystallographic data calculated with the program LAZY PULVERIX. A theoretical diffraction diagram calculated on this basis is shown in Figure 3.

Table 3 Diffraction peaks of the three materials and calculated intensities for assumed volume fractions of $69.5 \% \mathrm{AgBr}, 19 \% \mathrm{Ag}$ and $11.5 \% \mathrm{AgCL}$. Also is indicated which peaks were measured with sample to detector distances of $50 \mathrm{~cm}$ and $100 \mathrm{~cm}$ respectively.

\begin{tabular}{llllll}
\hline Phase & & & & $\begin{array}{l}\text { Relative } \\
\text { Intensities } \\
{[\%]}\end{array}$ & $\begin{array}{l}\text { Measuring } \\
\text { Distance } \\
{[\mathrm{cm}]}\end{array}$ \\
\hline $\mathrm{AgBr}$ & $(h k l)$ & $2 \vartheta$ & $F(h k l)$ & 0.8 & \\
$\mathrm{AgC1}$ & 111 & 23.01 & 0.3 & 2.0 & \\
$\mathrm{AgBr}$ & 111 & 23.96 & 1.4 & 96.4 & 100 \\
$\mathrm{AgC1}$ & 200 & 26.63 & 5.1 & 21.8 & 100 \\
$\mathrm{Ag}$ & 200 & 27.73 & 6.2 & 5.2 & 50 \\
$\mathrm{Ag}$ & 111 & 32.95 & 2.4 & 3.0 & 50 \\
$\mathrm{AgBr}$ & 200 & 38.24 & 2.4 & 100.0 & 50 \\
$\mathrm{AgC1}$ & 220 & 38.02 & 5.1 & 22.5 & \\
$\mathrm{AgBr}$ & 220 & 39.63 & 6.2 & 0.5 & \\
$\mathrm{AgC1}$ & 311 & 44.91 & 0.3 & 4.7 & \\
$\mathrm{AgBr}$ & 311 & 46.84 & 1.4 & 10.3 & \\
$\mathrm{AgC1}$ & 222 & 47.02 & 5.1 & 26.4 & \\
$\mathrm{AgBr}$ & 222 & 49.06 & 6.2 & 3.2 & \\
$\mathrm{Ag}$ & 400 & 54.86 & 5.1 & 6.0 & \\
$\mathrm{AgC1}$ & 220 & 55.18 & 2.4 & 6.2 & \\
\hline
\end{tabular}




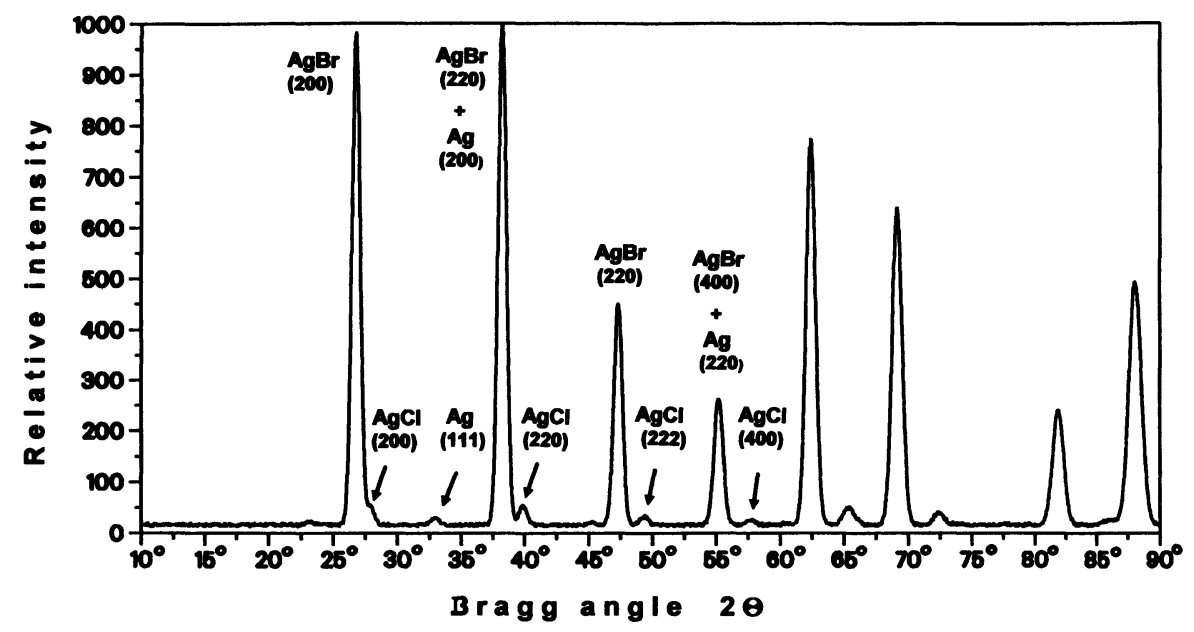

Figure 3 Theoretical diffraction diagram of a three-phase composite according to Table 3 .

Texture measurements were carried out using the neutron texture diffractometer TEX-2 at the research reactor FRG-1 in the Research Center GKSS at Geesthacht (Brokmeier 1994). In this instrument the flux at the sample position is $\sim 2.5 \cdot 10^{5}[n$ / $\mathrm{sec} \cdot \mathrm{cm}^{2}$ ]. The sample-to-detector distance is usually $50 \mathrm{~cm}$. In some cases, as is also indicated in Table 3 , it had to be increased to $100 \mathrm{~cm}$ in order to increase the angular resolving power. On the other hand, this decreases the count rate in the detector and thus requires still longer measuring times. Because of the non-axial sample symmetries, two-dimensional pole figures had to be measured (not only one-dimensional ones i.e. $\alpha$-scans, as is sufficient for true fibre textures). The measurements were done using an "equal-area" net as is shown in Figure 4 with measuring times per pole figure point of $3-5 \mathrm{~min}$ on a total of 679 points per pole figure.

The pole figures were used to calculate ODFs and inverse pole figures. Because of non-axial symmetry a computer program assuming triclinic sample symmetry was used in some cases. The calculations were done using the iterative series expansion method

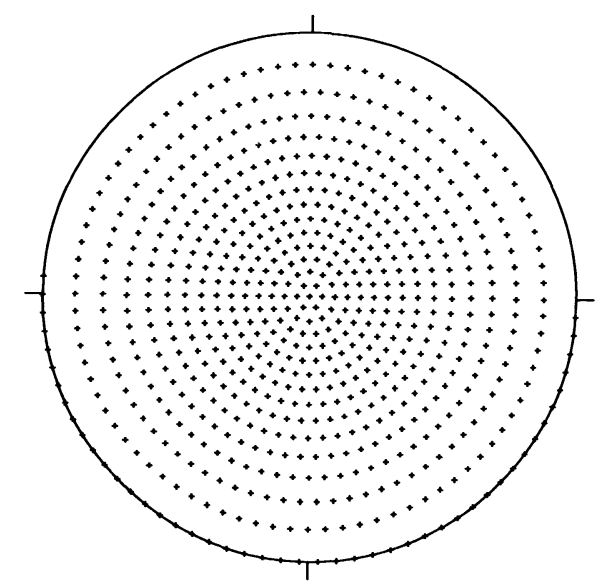

Figure 4 Equal area measuring net containing 679 points. 
Dahms and Bunge (1989), Dahms (1992)). This method includes the positivity condition for a chosen number of non-measured pole figures and the positivity condition for the ODF from which the odd part of the ODF is deduced. It is also possible to calculate the ODF's of two phases simultaneously from overlapped pole figures. This was the case for the $(200)_{\mathrm{Ag}}$ and $(220)_{\mathrm{AgBr}}$ reflection as is shown in Table 3.

For optical investigation the cross-sections of the fibre specimens were embedded in epoxy resin and after hardening grinded on $\mathrm{SiC}$ paper. After that, mechanical polishing with diamond paste was not suitable because of the softness of the material. Hence, a mechano-chemical polishing technique with $\mathrm{Na}_{2} \mathrm{~S}_{2} \mathrm{O}_{3}$ solution and polishing cloth was used. Finally, the surface of the samples was exposed to the light of the microscope for one or two hours which leads to visible contrasts between the phases.

\section{RESULTS}

In Figure 5a the cross-section of an extruded rod of $7.6 \mathrm{~mm}$ diameter is shown. One sees the non-circular interface between core and cladding as a result of non-axially symmetric flow in both materials leading also to non-axially symmetric textures. Another example is shown in Figure $5 \mathrm{~b}$ which corresponds to an extruded, cold drawn and annealed fibre of $3 \mathrm{~mm} \varnothing$. One sees the core, the initial cladding and a diffusion zone in between them as well as the outer Ag coating. In this case the interfaces are nearly circular. Furthermore, by annealing, the material had become coarse-grained as was also confirmed by texture measurements.

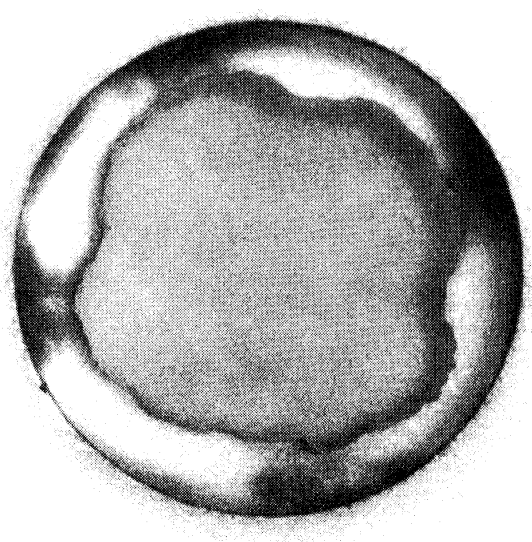

(a)

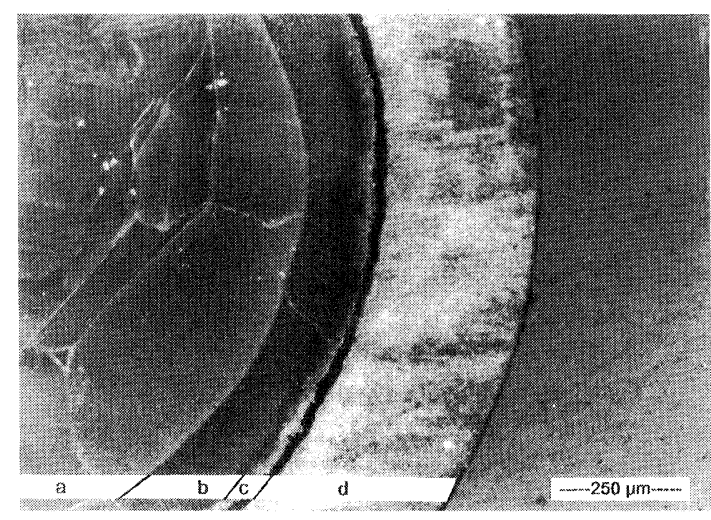

(b)

Figure 5 Micrographs of the cross-section of composite fibres

a) Extruded rod $7.6 \mathrm{~mm} \varnothing$

b) Cold drawn and annealed fibre $3 \mathrm{~mm} \varnothing$ (graded)
$\mathrm{a}=$ core
$\mathrm{b}=$ diffusion zone
$\mathrm{c}=$ initial cladding
$\mathrm{d}=$ coating 
The measured pole figures are given in Figure 6 for three typical samples i.e. an extruded two-phase sample $\mathrm{AgCl}+\mathrm{AgBr}$, a cold drawn coated sample $\mathrm{AgCl}+\mathrm{AgBr}$ $+\mathrm{Ag}$, and an annealed sample $\mathrm{AgCl}+\mathrm{AgBr}+\mathrm{Ag}$. This latter sample contains a diffusion zone $\mathrm{AgCl}-\mathrm{AgBr}$. The ODFs calculated from the pole figures are shown in Figure 7. They were obtained with a series expansion degreee of $L=23$. Finally, inverse pole figures are given in Figure 8.

\section{DISCUSSION OF THE RESULTS}

The extruded sample shows fairly axially symmetric textures both in the core and in the cladding (Figure 6a). Both textures are essentially double fibre textures with the directions $<111\rangle$ and $<100>$ parallel to the fibre axis (Figure 8). This is in good agreement with earlier studies of textures formed in this class of materials. In the AgClcladding the volume fraction of the $<111>$ component is much higher than that of the minor $<100>$ component. Also this is in agreement with former results. In the $\mathrm{AgBr}$ core, additionally a strong $<100>$ component is found. This must be attributed to the
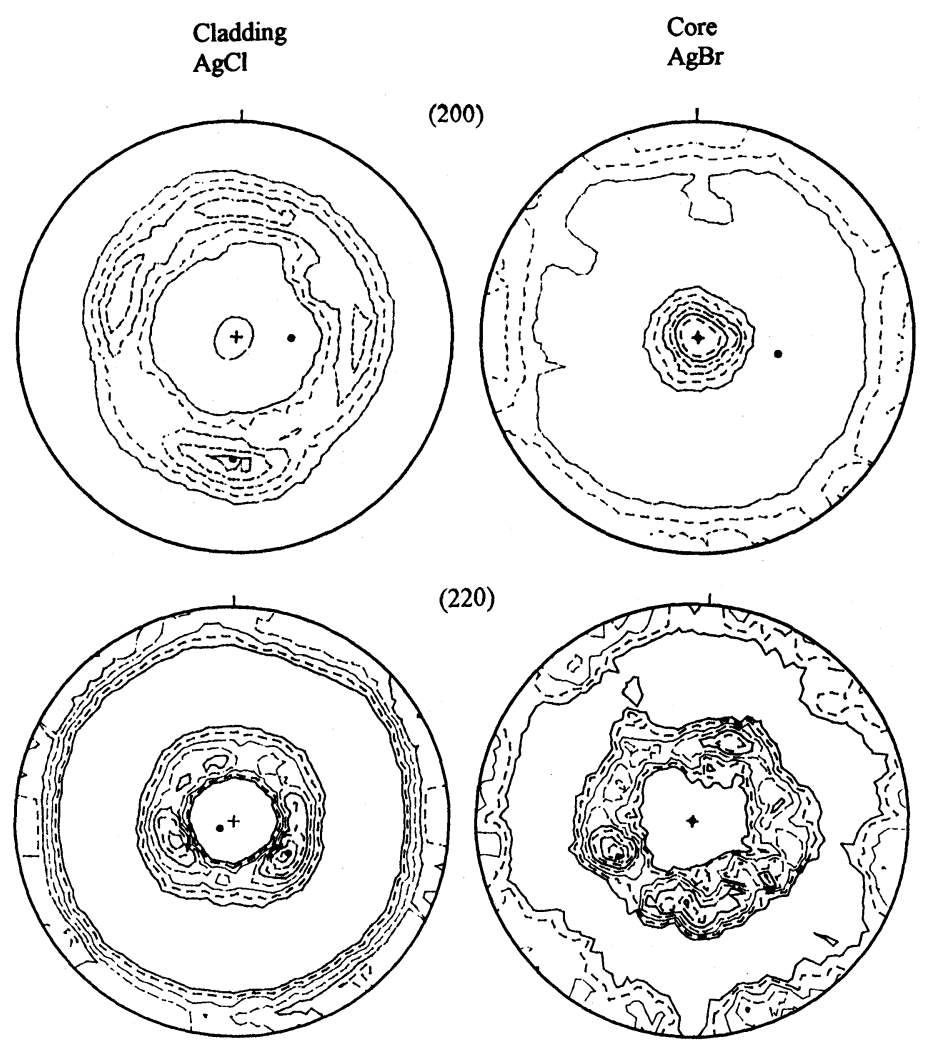

(220)

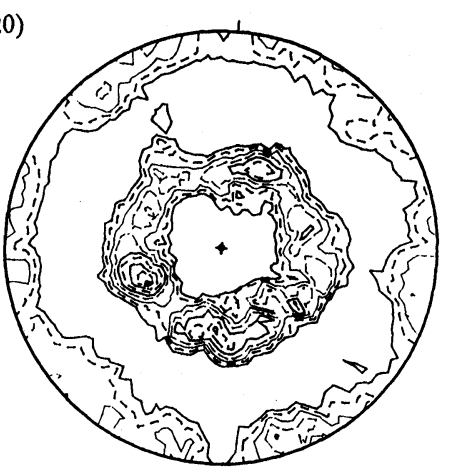

Figure 6(a) Pole figures of three composite samples according to Table 2 measured by neutron diffraction

- Extruded sample 

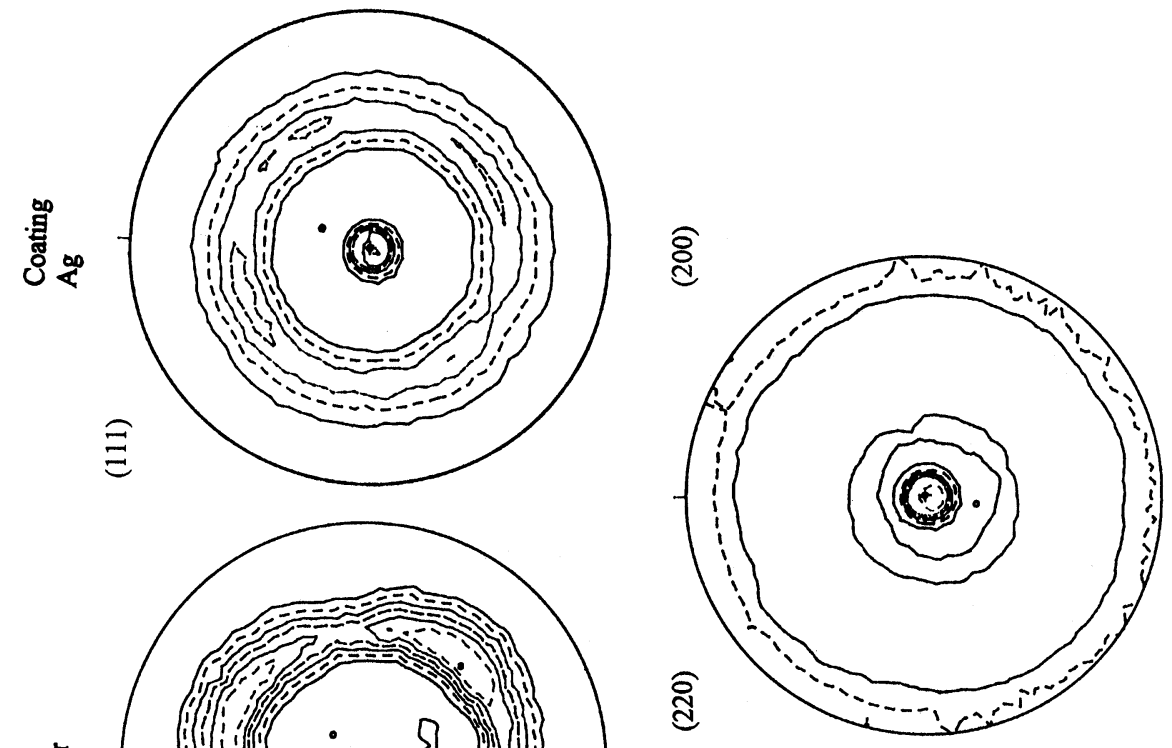

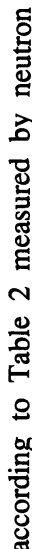
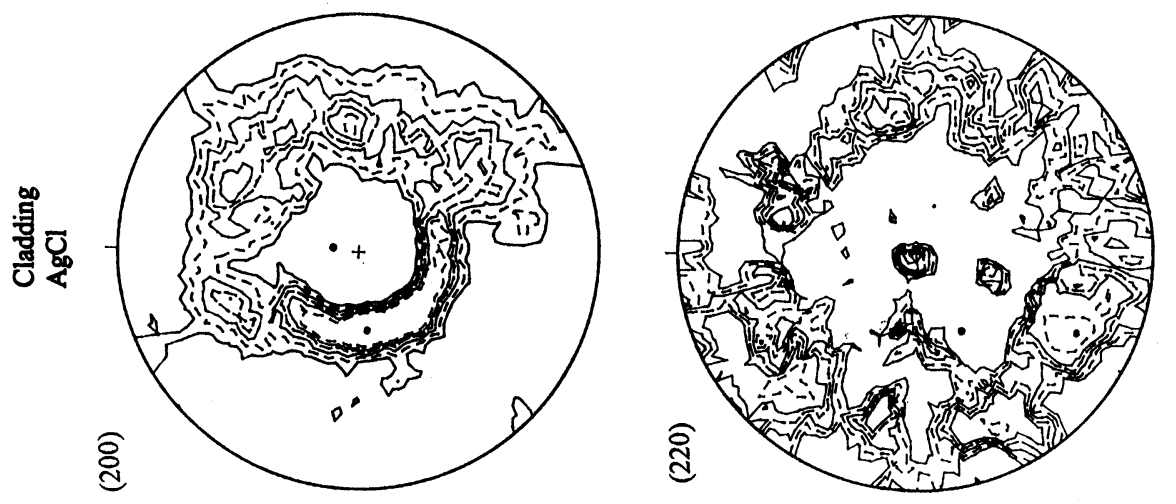

范 

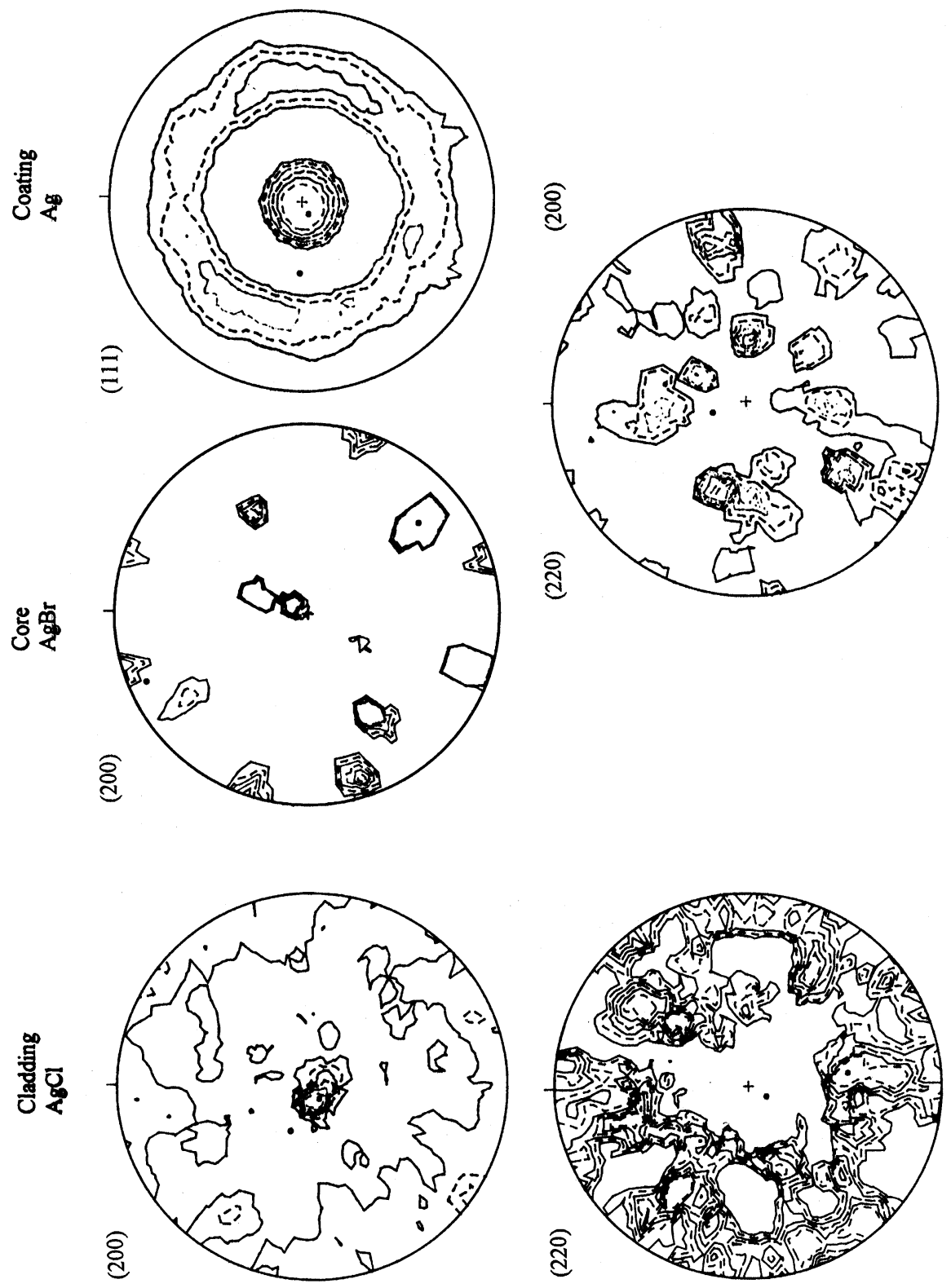

苍 
initial single crystal orientation of the core material which had a [100] - direction parallel to the extrusion direction. This original orientation can still be seen in the symmetry of the texture after extrusion (Figure 6a). It contains a component of four-fold symmetry which may be described as (001) [110] if we chose appropriate sample coordinate axes perpendicular to the fibre axis. (The relationship of these axes with the original single crystal axes was, however, not established). This component can also be seen in the ODF Figure 7 where it is superposed on the component of axial symmetry i.e. the

Extruded

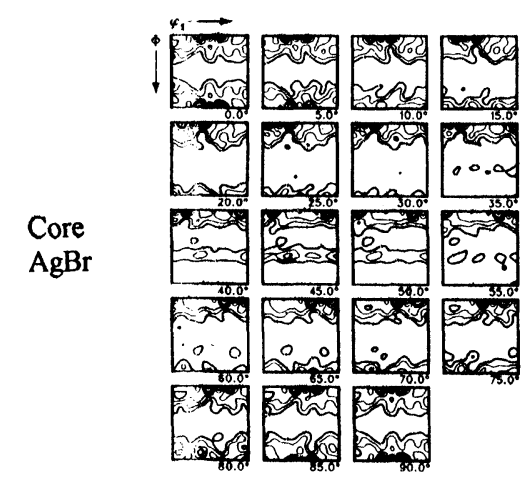

Cladding $\mathrm{AgCl}$

Coating

$\mathrm{Ag}$

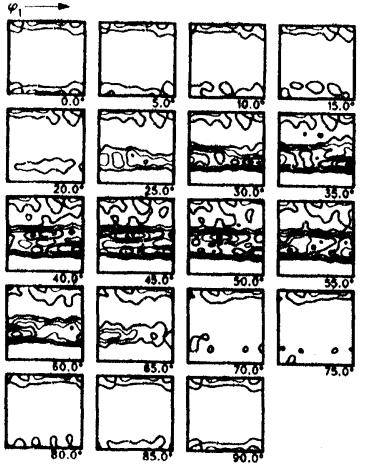

Cold drawn

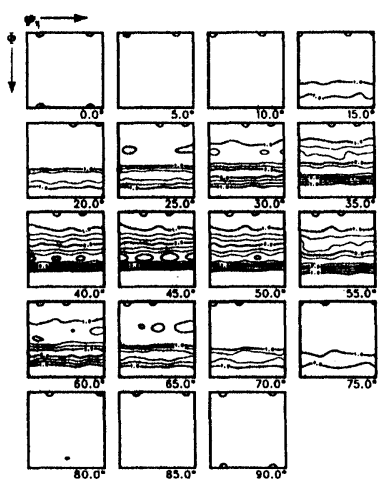

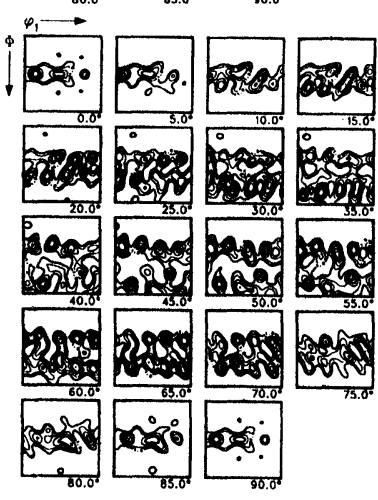
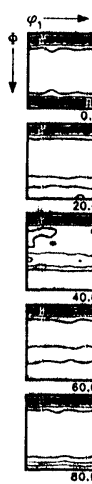
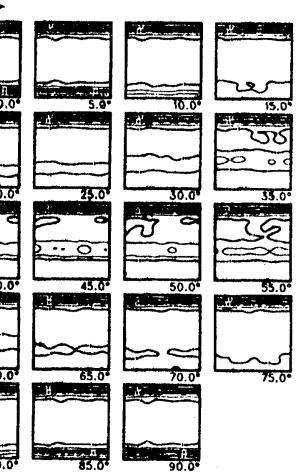

\begin{abstract}
Annealed
\end{abstract}
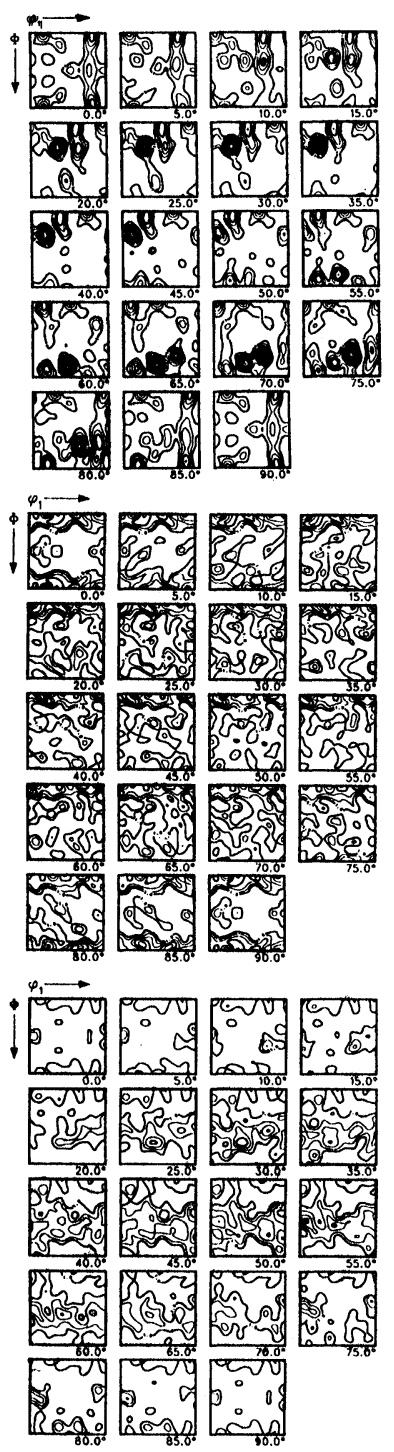

Figure 7(a) ODFs calculated from the pole figures

- Calculated with the assumption of orthorhombic sample symmetry 


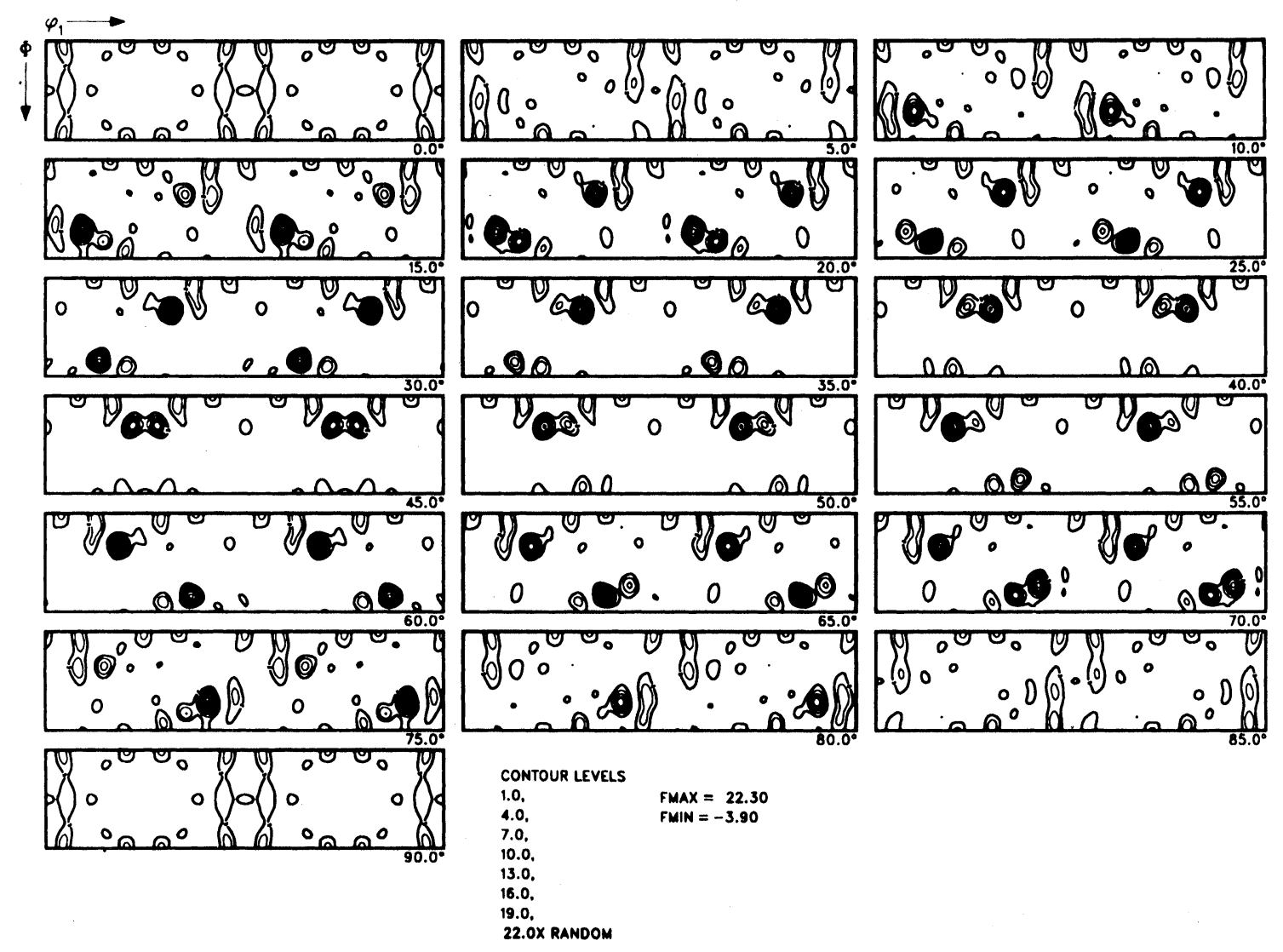

Figure 7(b) ODFs calculated from the pole figures

- ODF of the AgBr-phase of the annealed sample calculated with the assumption of triclinic sample symmetry

$<100>$ fibre texture component. A superposition of a single orientation on the $<111>$ fibre component is not to be expected and is not observed in the ODF. The $<111>$ - component is thus axially symmetric. It was formed out of the starting single crystal by orientation changes over long distances whereby the original crystal orientation can no more be seen.

The second investigated state corresponds to a sample which was extruded in the same way as the first one, put into a silver tube and then was cold drawn. (The extruded state of this sample was, however, not identical with the first sample). The $\mathrm{AgCl}$ cladding of this sample shows a very complex texture the interpretation of which is difficult. The non-axial symmetry of the pole figures Figure $6 \mathrm{~b}$ indicates non-axial flow during drawing. This may be in accordance with a near-circular interface between $\mathrm{AgCl}$ cladding and $\mathrm{AgBr}$. Because of the small thickness of the cladding a relatively small deviation of the interface from circular shape can cause a strong relative change of the wall thickness of the thin cladding. Furthermore, the "spotty" nature of the pole figures and even more so of the ODF 


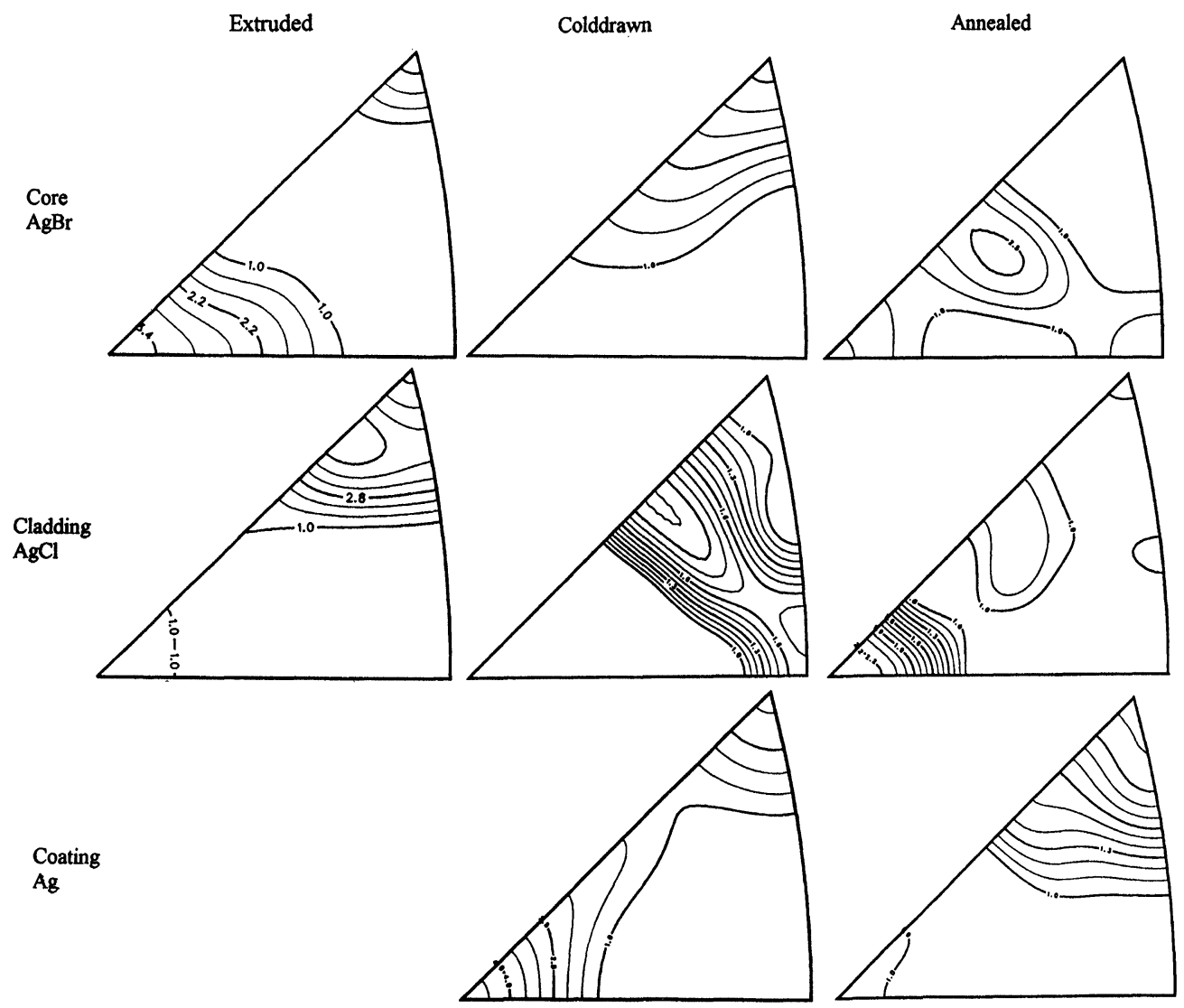

Figure 8 Inverse pole figures of the fibre axis direction

may be due to some recrystallization during (or shortly after) cold drawing leading to coarse grains. This was, however, not further checked. The $\mathrm{AgBr}$ core of this state shows only small deviations from axial symmetry. This is in accordance with a single crystalline preform which is now, however, deformed with a much higher total degree of deformation. The non-circular shape of the interface, assumed in the texture of the cladding, corresponds here to a much smaller relative deviation from axial symmetry of the core which has a much higher diameter. Also, the inverse pole figure (Figure 8 ) shows that the texture is mainly the $<111>$ fibre component expected in this class of material after high deformation degrees independent of the starting orientation. The initial texture of the Ag-tube before cold drawing was not measured. The texture of the cold drawn state is the double fibre texture $<111\rangle+<100\rangle$ (Figure 8 ) with a higher volume fraction of $\langle 100\rangle$ as has been frequently observed in $\mathrm{Ag}$ as a low stacking fault material. The texture of the Ag coating is very near to axial symmetry (Figure $6 b)$.

The third state, finally, corresponds to the same sample as that of the second but after additonal annealing $8^{h}$ at $400^{\circ} \mathrm{C}$. After this treatment recrystallization has taken place. This applies to all three phases, i.e. core, cladding and coating. The 
recrystallization texture of the cladding is essentially the $\langle 100\rangle-$ fibre texture. The "spottiness" of the pole figures indicates a coarse grain structure.The peaks on the $<100>$ fibre seen in the ODF may thus be attributed to the coarse grained nature of the sample. The core material shows a very strong spottiness in the pole figures. Hence, the ODF must be considered as consisting only of a non-representative selection of some big grains. The inverse pole figure shows that the orientations of these grains are in a broad range spread about $\langle 100\rangle$ extending towards $<211>$. This is the same tendency as observed in the cladding material. Finally, the Ag coating shows an axially symmetric recrystallization texture near to $<111\rangle$ in agreement with earlier investigations in $\mathrm{Ag}$ wires.

One of the questions to be answered by texture measurements in the present study was, if deviations from axially symmetric flow could be detected. Particularly, we are interested in a circular shape of the interface between core and cladding. This can be achieved by using a small diameter of the cladding. In this case even a small absolute deviation of the interface means a strong relative deviation of the cladding thickness and hence strong local texture changes in the cladding. This was assumed to be the reason for the non-axial symmetry of the cladding texture in the cold drawn state.

\section{CONCLUSIONS}

It was the purpose of the present investigation to find optimum conditions for the co-deformation of two- or three-layer composite samples, thereby retaining the original axial symmetry of the interface boundaries. Because of the different flow stresses of the materials of the different layers, plastic instabilities may occur which deform the interface boundaries into a non-circular shape. The problem is aggravated by the fact that at least the core material had to be single crystalline as a result of which non-circular anisotropy of the flow properties must be expected.

It could be shown that the absolute deviation of the core-cladding interface could be kept within acceptable limits although the local flow field in all phases may have deviated from axial symmetry. This should have lead to local texture fields in the phases. In the present investigation, only global textures of the composite were measured by neutron diffraction. The deviation of these textures from axial symmetry gives, however, good insight into the obtained flow conditions in all phases.

Texture measurements are also well suited to recognize the occurrence of recrystallization on the basis of the difference between deformation and recrystallization textures. Furthermore, the coarse grain structure after recrystallization can easily be seen. It must be concluded that even more detailed information about the flow process is to be expected from the measurement of local textures (i.e. texture fields).

\section{Acknowledgements}

The authors would like to thank GKSS-Research Centre Geesthacht GmbH for the support in neutron diffraction at the Research Reactor FRG - 1. This work was funded by the German Ministry for Research and Technology (BMFT) under the contract number 3BU3CLA F.4 - K19. 


\section{References}

Artjushenko, V. G. et al. (1986). Infrared polycrystalline waveguides made of silver halides. Sov. J. Quantum Elektron, 16, 3, 389.

Artjushenko, V. G. et al. (1991). Infrared Cables and Catheters for Medical Applications. SPIE, Vol. 1420 Optical Fibres in Medicine, VI/157.

Brokmeier, H. -G. (1994). Texture Analysis by Neutron Diffraction. Materials Science Forum, 157/162, 59-70.

Brokmeier, H. -G. Texturanalyse mittels winkeldispersiver neutronographischer Kernstreuung, GKSS - Report (to be published), 60-85.

Bunge, H. J. (1982). Texture Analysis in Materials Science, Butterworths Publ. London.

Dahms, M. (1992). The iterative series-expansion method for quantitative texture analysis, II. Applications. J. Appl. Cryst., 25, 258-267.

Dahms, M., Bunge, H. J. (1989) The iterative series-expansion method for quantitative texture analysis. I. General outline. J. Appl. Cryst., 22, 439-447.

Frommeyer, G., Wassermann. G., Verformungsverhalten von Aluminiumverbunddrähten mit Chloridzusätzen., Werkstofftechnik / J. Z. f. (1976) of Materials Technologie, 7, 136-138.

Nye, J. F. (1949). Plastic deformation of silver chloride. Proc. Roy. Soc London, A 198, $190-204$.

Sagel, K. (1958).Tabellen zur Röntgenstrukturanalyse. Springer Verlag Berlin. 